\title{
Elemental Mapping of Perovskite Solar Cells Using Multivariate
} Analysis: an Insight of Degradation Processes

\author{
Stefania Cacovich, ${ }^{\left[{ }^{[a]}\right]}$ Giorgio Divitini, ${ }^{[a]}$ Christopher Ireland, ${ }^{[a]}$ Fabio Matteocci, ${ }^{[b]}$ Aldo Di Carlo, ${ }^{[b]}$ and \\ Caterina Ducati ${ }^{[\mathrm{a}]}$
}

\begin{abstract}
The technology of perovskite-based solar cells is evolving rapidly, reaching power conversion efficiency certified values now exceeding $20 \%$. One of the main drawbacks hindering progress in the field is the long term stability of the cells: the mixed halide perovskites used in most devices are sensitive to humidity and degrade on a timescale varying from hours to weeks. The degradation mechanisms are poorly understood, but likely to arise from combined physical and chemical modifications at nanometer scale. The characterization of pristine and degraded materials is difficult due to their complex chemical and physical structure and their relatively poor stability. In this work we investigated the changes in local composition and morphology of a standard device after 2 months of air exposure in dark, using a scanning transmission electron microscope (STEM) for imaging and analysis with nanometer resolution. Thanks to a state of the art technique that combines STEM and energy dispersive X-ray spectroscopy (EDX), and the use of different decomposition algorithms for multivariate analysis we highlighted the migration of elements across the interfaces between the layers composing the device. We also noticed a morphological degradation of the hole transporting layer, representing one of the main factors enabling the infiltration of moisture in the device, which results in reduced performance.
\end{abstract}

\section{Introduction}

A quick evolution led perovskite solar cells to results competitive with established technologies [1], achieving both low cost and high power conversion efficiency [2], but still little is known about their chemical stability [3-6], which seems to be strongly influenced by the hygroscopic nature of the organic cation present in the unit cell [7] and by the quick thermal degradation of the organic hole transporting material currently employed $[8$ $9,10]$. The failure of the cells due to light, air exposure and temperature has been proved in previous works, showing a dramatic reduction of the photovoltaic parameters [11-14] within a few hundred hours. Recent studies have demonstrated that interface degradation is one of the main responsible for the loss of power conversion of the devices [15-16].

The degradation processes have been investigated using different characterisation methods, such as atomic force microscopy (AFM), scanning electron microscopy (SEM), ultraviolet-visible spectroscopy (UV/VIS), X-ray diffraction (XRD), time-of-flight secondary ion mass spectrometry (ToF-SIMS), grazing incidence small-angle X-ray scattering (GISAXS) [17-

[a] S. Cacovich, Dr. G. Divitini. Dr. C. Ireland, Dr. C. Ducati Department of Materials Science and Metallurgy University of Cambridge

27 Charles Babbage road, CB3 OFS, Cambridge, UK E-mail: sc82@cam.ac.uk

[b] Dr. F. Matteocci, Prof. A. Di Carlo

Department of Electronic Engineering

University of Rome "Tor Vergata", C.H.O.S.E. (Centre for Hybrid and Organic Solar Energy)

via del Politecnico 1, Rome 00133, Italy

Supporting information for this article is given via a link at the end of the document
19]. Although these techniques provide useful information about morphology, optical properties and crystal structure, they are not able to describe in detail the local elemental composition. This aspect is crucial in order to relate the macroscopic properties to the variation of the chemical structure and thus to the drop in performance of the devices.

\section{Results and Discussion}

In order to shed light on some of the mechanisms underlying the degradation processes of halide organic-inorganic perovskite based devices, we study the behaviour of an archetypical [20] unsealed device before and after two months of air exposure in dark at $22{ }^{\circ} \mathrm{C}$ and relative humidity $30 \%$. The morphology and the chemical composition of the interface between optically active layer and carrier transport layers play a key role in the operation of thin film devices [21-23], and can be studied at the nanoscale by analytical transmission electron microscopy (ATEM).

The analytical capabilities of the TEM have recently improved with the introduction of large-area silicon drift detectors (SDD) for energy-dispersive X-ray spectroscopy, which provide high collection efficiencies and can tolerate higher count rates than older Si (Li) versions [24]. This is particularly important for beamsensitive specimens, where the electron dose must be kept to a minimum to limit beam damage. To further enhance the EDX mapping capabilities of modern S/TEMs, we employ multivariate analysis [25], obtaining an improvement of the signal to noise ratio. Moreover, this novel quantification procedure naturally separates different compounds without introducing operator bias, and is particularly effective in the presence of complex compounds, such as those developed during solution-based perovskite synthesis in photovoltaic devices [26]

The solar cell under investigation was prepared according to a published method, involving double-step perovskite conversion [20]: fluorine doped tin oxide (FTO) glass was coated first by a compact (hole blocking) layer and then by a nanoporous $\mathrm{TiO}_{2}$ layer. The $\mathrm{TiO}_{2}$ scaffold was infiltrated and capped by methylammonium lead iodide. Spiro-OMeTAD (2,2',7,7'Tetrakis-(N,N-di-4-methoxyphenylamino)-9,9'-spirofluorene), acting as hole transporting layer, was spin coated on the perovskite layer; Au contacts were deposited on top. Focused ion beam (FIB) milling was used to extract cross sectional lamellae of the device [27], immediately after breaking the seal and after two months of air exposure, at room temperature in the dark. We prepared a second FIB specimen from the aged device - this was the preferred choice over re-examining the same lamella, since the ageing processes in a $2 \mathrm{D}$ system could not be representative of the ones happening in the device. The evolution of the morphology of the solar cell after two months of air exposure can be observed in the cross sectional views of Figure 1.a. The double step synthesis process produces a rough perovskite capping layer [28], with irregular pyramidal structures about $400 \mathrm{~nm}$ at the base and $250 \mathrm{~nm}$ in height. STEM highangle annular dark field (HAADF) images after two months of air exposure exhibit evident degradation of these structures that will be in investigated in more detail later on. 

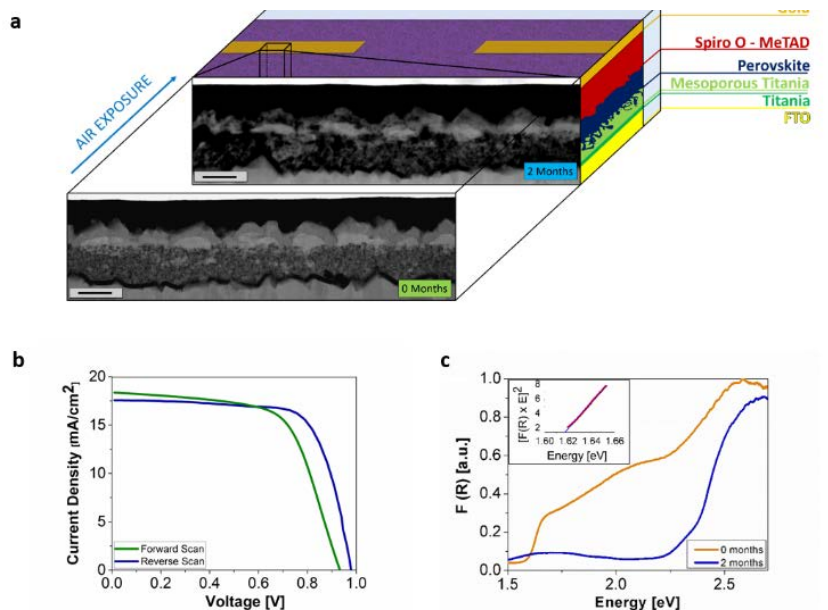

Figure 1. a) HAADF images and schematic of the device before and afte ageing. All scale bars are $400 \mathrm{~nm}$. b) Current -voltage curve of the fresh cell. c) Kubelka - Munk function calculated on 0 and 2 months old devices and transformed Kubelka Munk function measured on the diffuse reflectance spectrum at 0 months (inset).

The photovoltaic measurements of the device report a PCE (power conversion efficiency) of $11.9 \%$, with high $V_{o c}$ (opencircuit voltage) $956 \mathrm{mV}$ and $\mathrm{J}_{\mathrm{sc}}$ (short-circuit current) 17.47 $\mathrm{mA} / \mathrm{cm}^{2}$, as shown in the current voltage characteristics in Figure 1b.

Optical properties were investigated with reflectance measurements. We employed the Kubelka - Munk function, a method commonly used in diffuse reflectance spectrum analysis [29] to both gain information on the absorption of the material (by measuring the diffuse reflectance, and converting to absorbance via the Kubelka-Munk function) and to determine the nature (direct/indirect) of the allowed band-edge transition. Figure 1.c shows the Kubelka - Munk remission function related to reflectivity in both samples. The spectra appear clearly different. The pristine sample presents the well-known perovskite diffuse reflectance spectrum [30], while the contribution of the active layer disappears in the aged cells, where the spectrum is dominated by a peak at higher energy, around $2.6 \mathrm{eV}$, which can be associated to energy transitions from $\mathrm{Pbl}_{2}[31,32]$ and $\mathrm{TiO}_{2}$. To further investigate the optoelectronic properties of the device we report, in the inset in Figure 1.c, the transformed Kubelka - Munk function expressed as $[F(R) h u]^{2}$, with $h$ being Planck's constant and $u$ the wave frequency. From this graph we can determine the energy gap $\mathrm{E}_{\mathrm{g}}$ of the nanostructured composite cell to be $1.6 \mathrm{eV}$, and empirically proving that optical absorption takes place via a direct transition since the relation between the Kubelka - Munk function and the energy band gap follows a quadratic proportionality [33].

HAADF STEM images provide semi-quantitative information on the sample composition, since the contrast is proportional to the sum of the square of $Z$ numbers and to the thickness of the specimen under the beam [34]. We measured the intensity of the signal in the Z-contrast images acquired before and after ageing, averaging over equivalent areas within the various layers. We found a decrease of about $15 \%$ of signal intensity in the perovskite capping layer and in the scaffold layer, whereas no variations were observed in the Spiro- OMeTAD, FTO and gold layers. Lead and iodine being the heaviest elements in the system, and thus the ones that most affect the intensity of the image, we can conclude that there has been a loss of these elements.
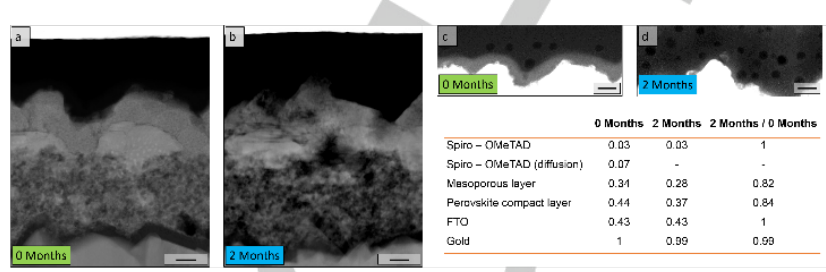

Figure 2. HAADF cross sectional view of the device before a) and after b) ageing. The contrast has been enhanced varying the dynamic range in figures c) and d) in order to investigate in more detail the HTM (hole transport material). All scale bars are $100 \mathrm{~nm}$. Table 1. Normalised average values (taking Au as reference) of the HAADF STEM intensity in the different layers.

Conversely, the contrast in the organic hole transport layer is significantly lower, and therefore the identification of small variations is complex. More information can be made clearer by heavily increasing the image contrast, as shown in Figure $2 \mathrm{c}, \mathrm{d}$ where we notice the presence of bubbles within the SpiroOMeTAD. The presence, and varying density, of such bubbles might affect the average HAADF STEM signal and cover chemical changes in the bulk of the hole transporter, so the data related to the hole transport layer intensity reported in Tab 1 have been measured in areas not containing bubbles. The increased contrast also makes another feature stand out: in the fresh sample a higher signal intensity, ascribed to the migration of inorganic elements from the perovskite layer [35], is visible close to the interface with the active layer (as shown in Fig 2c).

In Figure 3, where the HAADF contrast has again been enhanced to make the small variations in low brightness levels more visible, bubbles can be seen across the organic hole transport layer at lower magnification. We determined their density in terms of bubble/ $\mu \mathrm{m}^{2}$, and we notice an increase from a value of 24 bubbles $/ \mu \mathrm{m}^{2}$ to 62.4 bubbles $/ \mu \mathrm{m}^{2}$ upon ageing. The diameters range from 40 to $50 \mathrm{~nm}$ in the initial sample, comparable with AFM measurements reported in literature $[9,17]$, to a broader size distribution in the range of $20-70 \mathrm{~nm}$ in the aged one. We attribute the formation of these defects to the presence of dopants in the HTL and to the use of chlorobenzene as solvent $[9,36]$. The observation of this phenomenon is particularly important since it might be considered as one of the main reasons for the loss in performance of the cell, promoting the entrance of moisture and air within the device and resulting in a reduced active area for carrier transport [37].

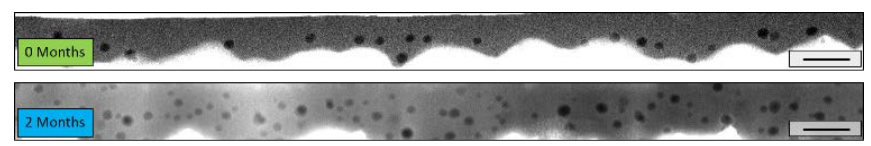

Figure 3. HAADF images at 0 (top) and 2 (bottom) months of the SpiroOMeTAD hole transport layer with increased contrast to highlight the presence of gas bubbles. All scale bars are $200 \mathrm{~nm}$.

STEM-EDX (Energy-Dispersive X-ray) analysis was carried out in order to investigate the local elemental variations occurring in the aforementioned device after two months of air exposure. EDX spectra were treated within Hyperspy [38], an open source Python library dedicated to hyperspectral data analysis, using a 
Principal Component Analysis (PCA) - based routine [39]. A model of the raw data has been reconstructed employing only the components containing physical information and discarding the noise-dominated ones (Figure $1 \mathrm{SI}$ ). On the denoised EDX spectra we performed Cliff-Lorimer quantification [40], obtaining elemental maps with $\mathrm{nm}$ scale - spatial resolution.

In Figure 4 comparative elemental maps of iodine, lead, carbon, oxygen and titanium of the two samples are shown. In the HAADF images the perovskite region appears split into two layers, with different signal intensity. EDX maps show a higher $\mathrm{Pb}$ and I content in the layer closer to the mesoporous $\mathrm{TiO}_{2}$, respectively 18 and 55 at. \% instead of 14 and $40 \%$ of the second perovskite layer. This can be related to the pyramidal shape of the top perovskite layer. As the pyramids get thinner towards the tip, the space around them - including the volume in front of and behind the perovskite - is filled with the hole transporter. The STEM-EDX signal originates through the entire thickness of the lamella (about $200 \mathrm{~nm}$ ), it therefore contains features from both the perovskite and the organic layer. This explains on one side the reduction of atomic percentage of the inorganic elements of the perovskite and on the other the higher percentage of carbon (Figure $4 \mathrm{~b}, \mathrm{c}, \mathrm{d}$ ). In the fresh sample the ratio between iodine and lead is equal to 3 , resulting almost constant in both the mesoporous and the capping regions. We remark a variation in the lead/iodine ratio only at the perovskite/HTL (hole transport layer) interface, where a higher amount of iodine brings the ratio to a value between 4 and 5 , confirming theoretical predictions about iodine migration as a first stage of degradation process $[41,42]$ and findings about the reactivity of oxidized Spiro-OMeTAD and iodide ions [43]. In the aged specimen the ratio between the two inorganic components has reduced to a value of 2.6, indicating a loss of iodine and the formation of $\mathrm{Pbl}_{2}$ or other intermediate compounds [44], as suggested by the optical measurements. In Figure 2 SI we report a specific compositional study aimed to identify the presence of heavy elements in the HTL. We compare the atomic percentage of iodine and lead in maps with increased contrast in order to identify also small variation in the chemical composition. lodine clearly diffuses in the HTL in the aged sample, whereas no migration of lead has been observed. In both samples, oxygen is present not only in the titania scaffold, but also in the perovskite layer, due to exposure to air during the synthesis process, as reported by previous ToF-SIMS studies [22]. Analysing the EDX spectra after the ageing process we can draw two main conclusions: iodine migration into the HTL can be observed, and carbon infiltrates into the pores left in the titania scaffold by the degradation of the perovskite component. In the
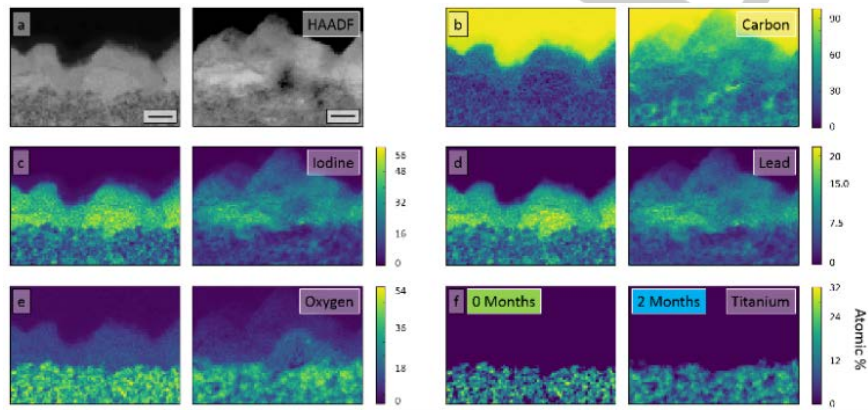

Figure 4. HAADF image (a) and elemental quantitative EDX maps before (left) and after (right) 2 months of air exposure for $\mathrm{C}$ (b), I (c), Pb (d), O (e) and $\mathrm{Ti}(\mathrm{f})$. All scale bars are $100 \mathrm{~nm}$.
In a similar fashion to applying PCA, Non-negative Matrix Factorisation (NMF), an alternative machine-learning algorithm widely employed in data processing [45], can be used to process the dataset. Thanks to constraints in the optimisation which are different from PCA, NMF can provide a decomposition of the spectra into factors that can be related to the chemical composition in a direct manner. It was thus applied on the fresh lamella dataset, resulting in a seven significant components, as shown in Figure 5a. Unlike the standard EDX quantification, this method allowed us to identify the different materials present in the sample as compounds rather than individual elements. In particular this method is suitable to accurately determine the grade of infiltration of the perovskite layer in the titania scaffold as evident by the representation of the components 1 and 2 in Figure $5 \mathrm{a}$. On the fresh sample the perovskite looks well infiltrated and the $\mathrm{TiO}_{2} /$ Perovskite interfaces appear sharp. The third component, associated to the HTL, shows the presence of small circular regions with reduced signal that can be physically interpreted again as gas bubbles in the Spiro-OMeTAD. These voids are likely to be introduced during the processing of the $\mathrm{HTL}$, and have been observed in the literature [17]. Interestingly a component that can be associated to iodine also appears, highlighting a more intense signal in the top part of the perovskite close to the Spiro-OMeTAD interface, and a diffusion into the HTL. NMF analysis was then carried out on a stack including maps of both the pristine and the aged device, so that both datasets were fitted with the same components. In Figure $5 \mathrm{~b}$ a component representing the inorganic part of the perovskite compound is illustrated, highlighting a correlation between $L_{a}$ and $M_{\alpha}$ lead peaks and the $L_{\alpha}$ iodine peak. Comparing the intensity of the signal coming from the infiltrated perovskite and the perovskite capping layer, we notice a reduction of about $50 \%$ after 2 months. The drastic reduction means that the stoichiometry has not been maintained constant during two months. In the aged cells a portion of the perovskite layer is still present in its original composition but ionic migration leads to the formation of $\mathrm{Pbl}_{2}$ nanoparticles, as evidenced by the optical measurements. A further evidence of the presence of these precipitates, although not directly observed by STEM images, is the reduction of the ratio $1 / \mathrm{Pb}$, previously discussed in EDX quantitative analysis, which can be ascribed to the overlapping of signal coming from both perovskite and $\mathrm{Pbl}_{2}$ particles. The chemical deterioration of the active layer results thus in a change of its absorption properties and finally in the macroscopic loss of performance of the cell. 

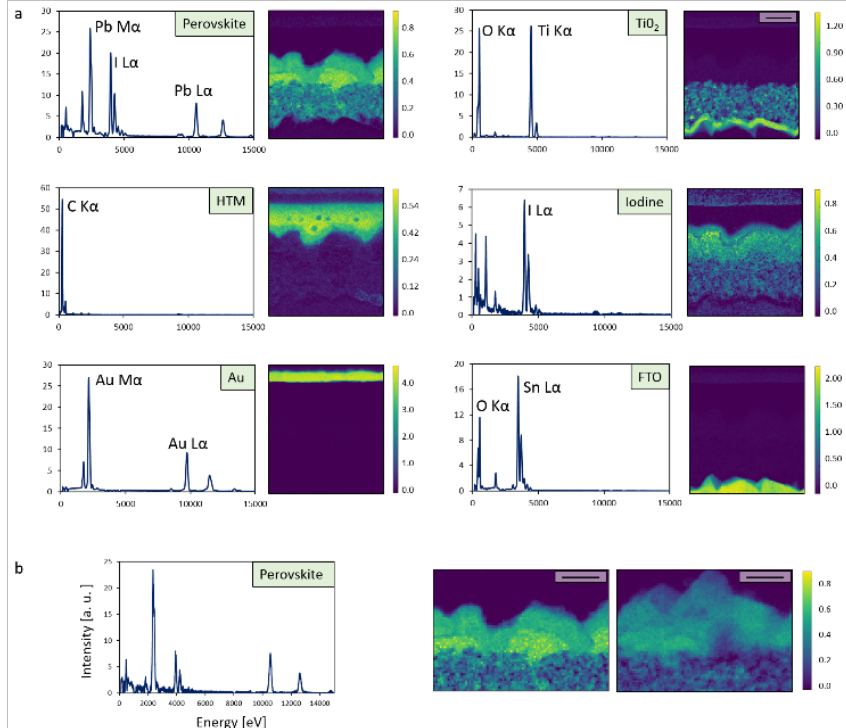

Figure 5. a) Non-negative Matrix Factorisation of the EDX map acquired on the freshly-prepared sample. Different compounds are isolated and identified from their EDX features; the loading of the corresponding component represents the spatial distribution of the component in the sample. b) NMF component and loading representing the perovskite layer of fresh and aged lamella. All scale bars are $200 \mathrm{~nm}$.

\section{Conclusions}

The device appears drastically and irreversibly damaged by air exposure. We proved the effects of ageing on physical properties of the cell using traditional macroscopic optical and photovoltaic characterization, moreover we identified different nanoscale processes that can contribute to the change in properties. The principal variations in local elementa composition and in morphology concern respectively the migration of iodine into the HTL layer towards the Au electrode, resulting in a severe degradation of the photoactive layer and the physical formation of bubbles in the Spiro-OMeTAD.

A quantitative understanding of the processes occurring upon air exposure in perovskite-based solar cells was reached by combining several cutting edge analytical transmission electron microscopy and data processing techniques, as well as optical measurements. In particular the use of FIB specimen preparation, combined with analytical transmission electron microscopy, is a powerful and versatile tool for the characterization of devices based on hybrid composites with nano- and micro-scale structural and chemical features. Furthermore the latest developments in TEMs and data processing open up new opportunities for the investigation of optoelectronic devices, including beam sensitive materials. In conclusion, the identification of such phenomena, for the first time shown at the nanoscale, will prove instructive for the design of the synthesis and operating procedures, improving the stability and enhancing the lifetime of the devices.

\section{Experimental Section}

Device fabrication and photovoltaic characterisation

Patterned FTO substrate (Pilkington, $8 \mathrm{ohms}$ per square) was used as transparent conductive oxide. Then a $\mathrm{TiO}_{2}$ compact layer (c- $\mathrm{TiO}_{2}, 80 \mathrm{~nm}$ thick) was deposited on a patterned FTO layer using Spray Pyrolysis Deposition following the procedure reported in literature [46]. A $250 \mathrm{~nm}$ thick mesoporous $\mathrm{TiO}_{2}$ layer (18NR-T paste, Dyesol, diluted with terpineol and ethylcellulose) was deposited by screen-printing and sintered at $480^{\circ} \mathrm{C}$ for $30 \mathrm{~min}$. The perovskite layer was deposited using a two-step method [47]. Lead iodide solution $\left(\mathrm{Pbl}_{2}\right.$ in $\mathrm{N}, \mathrm{N}$ dimethylformamide, Sigma-Aldrich, $330 \mathrm{mg} \mathrm{ml}^{-1}$ ) was deposited by airassisted blade-coating technique in air according to a previously reported procedure [19]. An air flow (speed: $17 \mathrm{~m}^{3} \mathrm{~s}^{-1}$, temperature: $100^{\circ} \mathrm{C}$ ) was applied to evaporate the solvent during the deposition. $\mathrm{A} \mathrm{Pbl}_{2}$ layer was annealed in air at $100^{\circ} \mathrm{C}$ for 1 hour. The perovskite layer was obtained by dipping in methylammonium iodide (MAl) solution for 30 minutes in air. 2,20,7,70-tetrakis-(N,N-dip-methoxyphenylamine)9,9'-spirobifluorene

(Spiro-OMeTAD, $75 \mathrm{mg} \mathrm{ml}^{-1}$ in chlorobenzene) was used as hole transport material and deposited by spin-coating (2000rpm, 40s) Prior to the spin-coating process the Spiro-OMeTAD was doped with $8 \mu \mathrm{l}$ of tertbutylpyridine and $12 \mu \mathrm{l}$ of lithium bis-(trifluoromethanesulfonyl) imide ( $\mathrm{Li}$ TFSI) solution ( $520 \mathrm{mg}$ in $1 \mathrm{ml}$ of acetonitrile). Finally, the samples, having an active area of $0.5 \mathrm{~cm}^{2}$ were transferred into a high vacuum chamber $\left(10^{-6} \mathrm{mbar}\right)$ to thermally evaporate the Au top contact $(100 \mathrm{~nm})$. Masked devices were tested using an aperture of $0.2500 \mathrm{~cm}^{2}$ under solar simulator (class A, SUNABET 2000) at AM1.5G and $100 \mathrm{~mW} \mathrm{~cm}$ illumination conditions.

Electron microscopy and data analysis

A FEI Helios Dual beam FIB/FEGSEM was employed to prepare lamellae for STEM imaging and analysis. EDX data were acquired using a FEI Tecnai Osiris TEM equipped with a high brightness Schottky XFEG gun and a Super-X EDX system composed by four silicon drift detectors, each approximately $30 \mathrm{~mm}^{2}$ in area and arranged symmetrically around the optic axis to achieve a collection solid angle of $0.9 \mathrm{sr}$. Spectrum images were acquired with a probe current of $0.7 \mathrm{nA}$, an acceleration voltage of $200 \mathrm{kV}$, a spatial sampling of $10 \mathrm{~nm} /$ pixel and 100 $\mathrm{ms} /$ pixel dwell time. Data were acquired with TIA and analysed with Hyperspy.

\section{Absorption measurements}

Diffuse reflectance measurements were carried out using an Ocean Optics setup: an ISP-REF integrating sphere was linked to a USB4000 UV-VIS-ES spectrophotometer by a $2 \mathrm{~m}$ long, $400 \mu \mathrm{m}$ core, solarisationresistant optical fiber, with the spectrum measured between $400-900$ $\mathrm{nm}$, and a PTFE reflectance standard. The spectra were analysed using the Kubelka-Munk function, $F(R)=(1-R)^{2} / 2 R=K / S$, with $R$ the reflectance, $\mathrm{K}$ the absorption and $\mathrm{S}$ the scattering coefficient, to represent the absorption coefficient of the sample. The direct band gap calculations were determined by plotting $(F(R) \times h u)^{2}$ vs $E$, with $F(R)$ determined from the reflectance as described.

\section{Acknowledgements}

S.C., G.D., C. I. and C.D. acknowledge funding from ERC under grant number 259619 PHOTO EM. C.D. acknowledges financial support from the EU under grant number 312483 ESTEEM2. F.M. and A.D.C. acknowledge funding from "Polo Solare Organico" Regione Lazio, the "DSSCX" MIURPRIN2010 and FP7 ITN "Destiny". S.C. and G.D. thank Dr. Francisco de la Peña and Dr. Pierre Burdet for assistance with PCA analysis. 
[1] M. Green, K. Emery, Y. Hishikawa, W. Warta, E.D. Dunlop, Prog. Photovolt: Res. Appl. 2015, 23, 1-9.

[2] H.J. Snaith J. Phys. Chem. Lett. 2013, 4, 3623-3630.

[3] M. Grätzel, Nature Materials 2014, 13, 838-842.

[4] N.-G. Park, Materials Today 2015, 18, 65-72.

[5] T. Leijtens, G.E. Eperon, N.K. Noel, S.V. Habisreutinger, A. Petrozza, H.J. Snaith, Adv. Energy Mater. 2015, 5, 1500963

[6] S.D. Stranks, H.J. Snaith, Nature Nanotech. 2015, 10, 391-402.

[7] J.H. Noh, S.H. Im, J.H., Heo, T.N. Mandal, S.I. Seok, Nano Lett. 2013 13, 1764-1769.

[8] T.A. Berhe, W.-N. Su, C.-H. Chen, C.-J. Pan, J.-H. Cheng, H.-M. Chen M.-C. Tsai, L.-Y. Chen, A.A. Dubale, B.-J Hwang, Energy Environ. Sci. 2016, 9, 323-356.

[9] L.K.Ono, S.R. Raga, M. Remeika, A.J. Winchester, A. Gabe, Y. Qi, J. Mater. Chem. A 2015, 3, 15451

[10] S. Ameen, M. Abdul Rub, S. A. Kosa, K. A. Alamry, M. Shaheer Akhtar, H.-S. Shin, H.-K. Seo, A. M. Asiri, M. K. Nazeeruddin, ChemSusChem 2016, 9, $10-27$.

[11] Y. Han, S. Mayer, Y. Dkhissi, K. Weber, J.M. Pringle, U. Bach, L. Spiccia, Y.B. Chen, J. Mater. Chem. A 2015, 3, 8139.

[12] B. Conings, J. Drijkoningen, N. Gauquelin, A. Babayigit, J. D'Haen, L. D'Olieslaeger, A. Ethirajan, J. Verbeeck, M. Manca, E. Mosconi, et al. Adv. Energy Mater. 2015, 5, 1500477.

[13] D. Bryant, N. Aristidou S. Pont, I. Sanchez-Molina T.Chotchunangatchaval, S. Wheeler, J. R. Durrant, S. A. Haque Energy Environ. Sci., 2016, 9, 1655

[14] K. Domanski, J.-P. Correa-Baena, N. Mine, M. K. Nazeeruddin, A Abate, M. Saliba, W. Tress, A. Hagfeldt, M. Gratzel ACS Nano, 2016, 10 (6), pp 6306-6314.

[16] A. Guerrero, J. You, C. Aranda, Y. Soo Kang, G. Garcia-Belmonte, H. Zhou, J. Bisquert, Y. Yang, ACS Nano 2016, 10, 218-224.

[17] Z. Hawash, L. K. Ono, S. R. Raga, M. V. Lee, Y. Q, Chem. Mater. 2015, 27, 562-569.

[18] J. Yang, B.D. Siempelkamp, T.L. Kelly, ACS Nano 2015, 9 [2], 19551963.

[19] J. Schlipf, P. Docampo, C.J. Schaffer, V. Körstgens, L. Bießmann, F. Hanusch, N. Giesbrecht, S. Bernstorff, T. Bein, P. Müller-Buschbaum, J. Phys. Chem. Lett. 2015, 6, 1265-1269.

[20] S. Razza, F. Di Giacomo, F. Matteocci, L. Cinà, A.L. Palma, S Casaluci, P. Cameron, A. D'Epifanio, S. Licoccia, A. Reale, A.; et al. ,J. Power Sources 2015, 277, 286-291.

[21] N. Marinova W. Tress, R. Humphry-Baker, M. Ibrahim Dar, V. Bojinov, S.M. Zakeeruddin, M.K. Nazeerudin, M. Grätzel, ACS Nano 2015, 9 , 4200-4209.

[22] F. Matteocci, Y. Busby, J.J. Pireaux, G. Divitini, S. Cacovich, C. Ducati A. Di Carlo, ACS Appl. Mater. Interfaces 2015, 7, 26176-26183.

[23] H. Zhou, Q. Chen, G. Li, S. Luo, T.-B. Song, H.-S. Duan, Z. Hong, J. You, Y Liu, Y. Yang, Science 2013, 345, 542-546.

[24] R. Terborg, M. Rohde, Microsc. Microanal. 2003, 9, 120-121.

[25] C.M. Parish, L.N. Brewer, Ultramicroscopy 2010, 110, 134-143.
[26] D. Rossouw, P. Burdet, F. de la Peña, C. Ducati, B.R. Knappet, A.E.H. Wheatley, P.A. Midgley, Nano Lett. 2015, 15, 2716-2720.

[27] R.M. Langford, M. Rogers, Micron 2008, 39, 1325-1330.

[28] H.S. Ko, J.W. Lee, N.G. Park, J. Mater. Chem. A, 2015, 3, 8808.

[29] S.P. Tandon, J.P.; Gupta, Phys. Status Solidi 1970, 38, 363-367.

[30] J. Qiu, Y. Qiu, K. Yan, M. Zhong, C. Mu, H. Yan, S. Yang, Arrays. Nanoscale 2013, 5, 3245

[31] D.H. Cao, C.C. Stoumpos, C.D. Malliakas, M.J. Katz, O.K. Farha, J.T Hupp, M.G. Kanatzidis, APL Mater., 2014, 2, 091101.

[32] J.A. Christians, P.A. Herrera, P.V. Kamat, J. Am. Chem. Soc., 2015 137, 1530-1538

[33] H.-S.Kim, C.-R. Lee, J.-H. Im, K.-B. Lee, T. Moehl, A. Marchioro, S.-J. Moon, R. Humphry-Baker, J.-H. Yum, J.E. Moser, et al., Sci Rep. 2012 $2,591$.

[34] P.D. Nellist, S.J. Pennycook, Adv. Imaging Electron Phys. 2000, 113 147-203

[35] G. Divitini, S. Cacovich, F. Matteocci, L. Cinà, A. Di Carlo, C. Ducat Nature Energy, 2016, 1, 15012

[36] G.-W. Kim, G. Kang, J. Kim, G.-Y. Lee, H. II Kim, L. Pyeon, J. Lee, T. Park, Energy Environ. Sci. 2016, 9, 2326-2333.

[37] G.E. Eperon, S.N. Habisreutinger, T. Leijtens, B.J. Bruijnaers, J.J. van Franeker, D.W. deQuilettes, S. Pathak, R.J. Sutton, G. Grancini, D.S Ginger, D. S.; et al., ACS Nano 2015, 9, 9380-9393.

[38] F de la Peña, P. Burdet, M. Sarahan, T. Ostasevicius, J. Taillon, A Eljarrat, S. Mazzucco, V.T. Fauske, G. Donval, L.F. Zagonel, et al Hyperspy 0.8: Hyperspectral data analysis toolbox, doi: 10.5281/zenodo.16850.2015.

[39] S. Wold, K. Esbensen, P. Geladi, Chemometrics Intelligent Lab. Syst 1987, 2, $37-52$.

[40] G. Cliff, G.W. Lorimer, J. Microsc. 1975, 103, 203-207.

[41] J.M. Azpiroz, E. Mosconi, J. Bisquert, F. De Angelis, Energy Environ. Sci. 2015, 8, 2118-2127.

[42] C. Eames, J.M. Frost, P.R.S. Barnes, B.C. O'Regan, A. Walsh, M. Saiful Islam, Nature Commun. 2015, 6, 7497.

[43] J. Carrillo, A. Guerrero, S. Rahimnejad, O. Almora, I. Zarazua, E. MasMarza, J. Bisquert, G. Garcia-Belmonte, Adv. Energy Mater. 2016, 6 1502246.

[44] S. Rahimnejad, A. Kovalenko, S. Martí Forés, C. Aranda, A. Guerrero, ChemPhysChem 2016, 17, 1 - 5 .

[45] V.P. Pauca, J. Pipera, R.J. Plemmons, Linear Algebra and its Applications 2006, 416, 29-47.

[46] F. Matteocci, G. Mincuzzi, F.Giordano, .A Capasso, E. Artuso, C. Barolo, G. Viscardi, T.M. Brown, A. Reale, A. Di Carlo, Org. Electron 2013, 14, 1882- 1890.

[47] J. Burschka, N. Pellet, S.-J. Moon, R. Humphry-Baker, P. Gao, M. K Nazeeruddin, M. Grätzel, Nature 2013, 499, 316-319. 
Entry for the Table of Contents (Please choose one layout)

Layout 1:

\section{FULL PAPER}

Perovskite chemical nanoscale characterisation: Some of the mechanisms underlying the loss in performance of halide organicinorganic perovskite have been studied using STEM EDX techniques and employing multivariate analysis. We highlighted the migration of elements across the interfaces between the layers composing the device and a morphological degradation of the hole transporting layer
Author(s), Corresponding Author(s)*

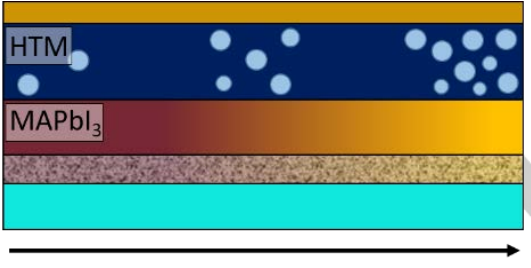

Page No. - Page No.

Title

Time 